Itinéraires Itinéraires

Littérature, textes, cultures

\title{
Douceur de la vengeance : portraits masculins dans Ourika de Claire de Duras
}

\section{Mary Donaldson-Evans}

\section{(2) OpenEdition}

\section{Journals}

Édition électronique

URL : http://journals.openedition.org/itineraires/2215

DOI : $10.4000 /$ itineraires.2215

ISSN : 2427-920X

Éditeur

Pléiade

\section{Édition imprimée}

Date de publication : 1 décembre 2008

Pagination : 121-133

ISBN : 978-2-296-07519-1

ISSN : 2100-1340

Référence électronique

Mary Donaldson-Evans, «Douceur de la vengeance : portraits masculins dans Ourika de Claire de Duras », Itinéraires [En ligne], Numéro inaugural | 2008, mis en ligne le 01 décembre 2008, consulté le 19 avril 2019. URL : http://journals.openedition.org/itineraires/2215; DOI : 10.4000/itineraires.2215

\section{(C) $(\oplus \Theta$}

Itinéraires est mis à disposition selon les termes de la licence Creative Commons Attribution - Pas d'Utilisation Commerciale - Pas de Modification 4.0 International. 


\title{
Douceur de la vengeance: portraits masculins dans Ourika de Claire de Duras
}

\begin{abstract}
This essay argues that Claire de Duras's Ourika can be seen as a somewhat acrimonious reply to Chateaubriand's Atala. Together with certain narrative, structural, and thematic elements shared by the two works, the role that falls to male characters is noteworthy. In opposition to the magnanimous heroes depicted by her forebear, Duras's male characters are defined by a failure of reason that makes them incapable of heroism.
\end{abstract}

L'amitié qui unissait Claire de Duras et René de Chateaubriand est bien connue. Bien connu aussi le caractère inégal de cette amitié. S'étant rencontrés en 1808, aristocrates ayant tous deux fait l'expérience de l'exil pendant la Révolution, ils avaient tout pour s'entendre, et l'engouement de $\mathrm{M}^{\mathrm{me}} \mathrm{de}$ Duras pour son aîné était d'autant plus complet que son mariage avec le duc de Duras était décevant. Toujours est-il que c'est ce même mariage avec un maréchal de France qui lui a permis de secourir Chateaubriand pendant la Restauration, prenant son parti contre Louis XVIII qui lui était hostile', obtenant pour lui des postes diplomatiques et lui prêtant parfois de l'argent. Chateaubriand, pour sa part, " une coquette [avec] un petit sérail ${ }^{2}$ » selon $\mathrm{M}^{\mathrm{me}}$ de La Tour du Pin, une des amies de $\mathrm{M}^{\mathrm{me}}$ de Duras, était trop occupé par son travail, sa femme malade et ses maitresses, pour venir en aide à celle qu'il appelait sa « sœur» quand elle avait besoin de lui. Déjà consciente de l'égoïsme de son «tyrannique enfant gâté » en 1821, l'année où elle écrivit Ourika, $\mathrm{M}^{\mathrm{me}}$ de Duras allait donner libre cours à sa rancune dans une lettre à Chateaubriand rédigée deux ans plus tard :

1. Voir $\mathrm{M}^{\mathrm{me}}$ de Duras, Ourika. Édouard. Olivier ou le secret, Paris, Gallimard, coll. « Folio Classique », 2007, préface de Marc Fumaroli. Toute référence ultérieure au roman sera puisée dans cette édition.

2. G. Pailhès, La Duchesse de Duras et Chateaubriand, Paris, Perrin et Cie, 1910, p. 103. 
Quand je [...] pense que depuis quinze ans, je préfère ce qui est vous à ce qui est moi, que vos intérêts et vos affaires passent mille fois avant les miennes, et cela tout naturellement, sans que j'y aie le moindre mérite, et que je pense que vous ne feriez pas le plus léger sacrifice pour moi, je m'indigne contre moi-même de ma folie ${ }^{3}$.

Un tel passage, loin d'être isolé dans la correspondance de $\mathrm{M}^{\mathrm{me}}$ de Duras, invite à une lecture biographique d'Ourika - lecture que G. Pailhès a proposée en 1910. Interprétant l'ouvrage de Duras comme un roman à clef inspiré en grande partie par l'absence de réciprocité dans son amitié avec Chateaubriand, l'abbé Pailhès ne manque pas de perspicacité, mais il sousestime la finesse de Duras. La misogynie de son jugement («Les œuvres de femmes sont-elles souvent autre chose que des réminiscences et des souvenirs? » [p. 29]) appelle une correction.

«Une Atala de salon. » Cette caractérisation d'Ourika, personnage éponyme du roman publié par Duras en 1823, est l'invention de Louis XVIII (à moins qu'il n'ait dit «Un Atala de salon », selon la version rapportée par l'éditeur des Mémoires de la marquise de La Tour ${ }^{4}$ ). S'agit-il d'une comparaison entre deux ouvrages ou deux personnages? C'est la question que se pose Roger Little dans son excellente édition d'Ourika, et que nous reposons à notre tour. Mise à part l'intention apparente de dénigrer le talent de $\mathrm{M}^{\mathrm{me}}$ de Duras en comparant son œuvre à celle d'un homme qu'il respectait peu, le commentaire du roi vaut la peine qu'on s'y arrête. Alors que plusieurs critiques ont vu un lien entre René et Ourika ${ }^{5}$, personne, à ma connaissance, n'a songé à relever le gant jeté par Sa Majesté. Malgré la filiation suggérée par la ressemblance des titres - Ourika, comme Atala, étant un nom exotique marqué par le féminin -, la disparité entre les deux romans semble énorme. Ourika et René, pourtant, traitant de l'expérience de l'exil au moyen de fines analyses psychologiques, présentent de nombreux parallèles, et ce à partir même de l'épigraphe de Byron ( «This is to be alone, this, this is solitude! ») qui figure à la première page d'Ourika et qui encourage le lecteur à rapprocher l'histoire racontée par Chateaubriand de celle de Duras ${ }^{6}$. Entre Atala et Ourika, les liens sont plus subtils, mais mon but sera de montrer que le second peut être lu comme une mordante réplique au premier.

3. Cité par Pailhès, op. cit., p. 14.

4. Voir C. de Durfort, duchesse de Duras, Ourika, présentation et étude de R. Little, Exeter, University of Exeter Press, 1998, p. 101.

5. Cf. K. Weil, «Romantic Exile and the Melancholia of Identification », Differences: A Journal of Feminist Cultural Studies, $\mathrm{n}^{\circ}$ 7, 2, 1995, p. 111-125.

6. Le vers de Byron, remis dans son contexte, évoque la distinction entre le sentiment d'isolement ressenti par celui qui se trouve seul dans la Nature (tel René) et la solitude bien plus profonde et pénible de celui qui se sent seul au milieu des foules: c'est la situation d'Ourika. La citation vient du deuxième chant du Pèlerinage de Childe Harold; cf. édition citée en note 4, p. 50. 
Dans quelle mesure la juxtaposition proposée par le roi est-elle justifiée? Pour répondre à cette question, je commencerai par esquisser quelques ressemblances entre les deux petits romans, pour me pencher ensuite sur les portraits masculins dans Ourika. Mettant en question les stéréotypes masculins de l'époque, stéréotypes pleinement assumés dans Atala, ces portraits fournissent la clef d'une nouvelle lecture d'un roman qui a laissé Chateaubriand, son premier lecteur, « tout ému ${ }^{7}$ ». Ainsi, il ne s'agira ni de faire l'inventaire des attitudes racistes exposées dans le roman de $\mathrm{M}^{\mathrm{me}} \mathrm{de}$ Duras ni de faire de l'exil de l'héroïne une analyse strictement féministe. D'excellentes études inspirées par ces préoccupations existent déjà, et la lumière qu'elles ont jetée sur les coins les plus obscurs du roman fait qu'il ne reste guère d'ombres à éclairer ${ }^{8}$.

« Les amours de deux sauvages dans le désert » : à lui-même, le soustitre d'Atala suggère une opposition. Chez Duras, il s'agit non d'amour partagé mais de solitude, non de deux «sauvages » (amérindiens) mais d'une seule «sauvage civilisée » (africaine), non de désert mais de ville. L'exotisme d'Ourika, loin d'être centré sur le lieu où l'histoire se déroule afin de produire un effet de dépaysement, s'incarne dans le personnage principal ${ }^{9}$. Si, dans Atala, la couleur locale est fournie par la description minutieuse des beautés de la nature américaine et par les allusions aux coutumes pittoresques des Amérindiens, chez Duras le concept s'enrichit d'un sens cruellement ironique: la couleur locale, c'est Ourika elle-même. Le personnage éponyme, ayant perdu sa mère, est « rapporté » du Sénégal à l'âge de deux ans par M. le chevalier de B., gouverneur de la colonie, qui la donne en cadeau à sa tante. Échappée à une vie d'esclave, la petite Noire connaîtra le bonheur d'une enfance privilégiée dans un milieu aristocratique jusqu'au moment où, grâce à une conversation entendue par hasard entre sa « bienfaitrice » $\mathrm{M}^{\mathrm{me}}$ de $\mathrm{B}$. et une de ses amies, la marquise de..., elle prend conscience de sa négritude et de l'impasse de sa condition: la couleur de sa peau la vouera à jamais à la solitude, car dans la société qui est devenue la sienne, «[q]ui voudra jamais épouser une négresse?» (p. 71). Ourika a beau rêver d'un avenir meilleur où, suivant le « grand

7. F. R. de Chateaubriand, Correspondance générale, Paris, Gallimard, 1983, t. IV, p. 233. 8. Voir les introductions de J. DeJean et de M. Waller, C. de Duras, Ourika, New York, Modern Language Association, 1994; C. Herrmann, introduction à l'édition féministe d'Ourika, Paris, Des femmes, 1979; C. Bertrand-Jennings, «Problématique d'un sujet féminin en régime patriarcal: Ourika de $\mathrm{M}^{\mathrm{me}}$ de Duras », Nineteenth-Century French Studies, $\mathrm{n}^{\circ}$ 23, 1-2, automne-hiver 1994-1995, p. 42-58; M. Chilcoat, «Confinement, the Family Institution, and the Case of Claire de Duras's Ourika », L'Esprit créateur, n 38, 3, 1998, p. 6-16; D. O'Connell, «Ourika: Black Face, White Mask », French Review, nº 47, numéro spécial, 6, printemps 1974, p. 47-56; N. Wing, Between Genders: Narrating Difference in Early French Modernism, Newark (Del.), University of Delaware Press, 2004.

9. G. Critchfield nie un « effet d'exotisme » dans Ourika. Son jugement est pertinent, pourvu qu'on entende par cette expression un " effet de dépaysement»; voir Three Novels of Madame de Duras : « Ourika », «Edouard», « Olivier », Paris, Mouton, 1975, p. 53. 
désordre » de la Révolution, elle serait «moins étrangère ». Bien instruite, cultivée, elle ne peut pas non plus retourner en Afrique vivre parmi les gens de sa race. Elle finira par entrer au couvent, car pour Dieu « il n'y a ni [...] nègres ni blancs » (p. 94). Hélas, malgré l'intervention d'un médecin, à qui elle raconte son histoire (et qui est le narrateur du récit-cadre), c'est trop tard: géographie et ethnicité (lieu et couleur) ont déjà conspiré à miner sa santé, et le danger est inévitable. Duras fait preuve ici d'une interprétation originale du concept de « couleur locale », dont l'exploitation est associée surtout à Chateaubriand. Au Meschacebé (nom amérindien du Mississippi) d'Atala, "moins cadre que personnage ${ }^{10}$ », succède Ourika, identifiée au fleuve du Maroc du même nom et assimilée au continent de sa naissance (« je devais représenter l'Afrique » [p. 69], dit-elle en parlant de son rôle dans un bal donné par $\mathrm{M}^{\mathrm{me}}$ de $\mathrm{B}$.).

Duras n'a pas inventé l'histoire d'Ourika de toutes pièces. Par $\mathrm{M}^{\text {me }}$ de La Tour du Pin, elle avait pris connaissance de l'histoire du chevalier de Boufflers, gouverneur du Sénégal, qui avait rapporté d'Afrique plusieurs cadeaux, parmi lesquels un perroquet vert, deux petits singes, un cheval, et pour sa tante, la princesse de Beauvau, « une petite captive» du nom d'Hourica. La petite avait, disait-on, des sentiments tendres pour le petitfils de $\mathrm{M}^{\mathrm{me}}$ de Beauvau. Elle est morte, on ne sait de quoi, en 1799, à l'âge de seize ans. Voilà la matière brute dont Duras a construit son roman.

Quand Duras s'est mise à écrire Ourika, Atala en était à sa douzième édition et avait inspiré de nombreux hypotextes et tout un commerce de souvenirs ${ }^{11}$. Se servant de la même forme classique (le récit encadré) mais adoptant un style sobre aux antipodes du lyrisme romantique de son aîné, Duras a emprunté à l'intrigue d'Atala ses données les plus élémentaires: un «sauvage civilisé » raconte à la première personne l'histoire de sa vie. Dans le récit imaginé par Chateaubriand, il s'agit d'un Amérindien, Chactas, qui, ayant perdu tout jeune un parent (en l'occurrence un père), se voit sauvé de l'esclavage certain par un « bienfaiteur » (l'Espagnol Lopez) et connaît ensuite, après une brève période de bonheur, une vie pleine de souffrances, parmi lesquelles la douleur d'un amour interdit et inassouvi avec une « sœur ». Résumée de cette façon, l'intrigue présente des parallèles évidents avec Ourika. Ces parallèles ne sont que superficiels. Entre les deux personnages féminins, Atala et Ourika, en revanche, il y a des symétries si frappantes qu'on ne saurait les attribuer au hasard. En dépit de la perspective masculine adoptée par Chateaubriand, l'intérêt principal de son roman réside dans le drame du personnage éponyme. Tout comme Ourika, Atala est victime d'une opération de sauvetage qui tourne mal. Née

10. F. R. de Chateaubriand, Atala, René, éd. D. Jullien, Paris, Nathan, 1990, p. 22.

11. Voir M. Waller, «Being René, Buying Atala: Alienated Subjects and Decorative Objects in Postrevolutionary France ", dans Rebel Daughters: Women and the French Revolution, dir. S. E. Melzer et L. W. Rabine, New York, Oxford University Press, 1992, p. 157-177. 
d'une union illicite, Atala vient au monde « dans le malheur ${ }^{12} »$. Pour sauver la vie de son enfant malade, sa mère promet à la Vierge que sa fille lui consacrera sa virginité si elle est épargnée. Atala survit et, à l'âge de seize ans, prend conscience du serment de sa mère quand celle-ci, se trouvant à la dernière extrémité, la conjure de le respecter: «si tu ne tiens ma promesse, tu plongeras l'âme de ta mère dans des tourments éternels. » (p. 137) La chasteté ne lui pèse qu'à partir du moment où elle rencontre Chactas, qu'elle sauve d'entre les mains des Muscogulges. Fuyant à travers le désert, les deux jeunes Amérindiens tombent amoureux l'un de l'autre et sont sur le point de s'abandonner à leur passion quand un orage éclate. Survient le père Aubry, missionnaire qui les « sauvera » en les amenant dans sa mission. Là, Atala meurt, empoisonnée de sa propre main pour ne pas rompre le serment de sa mère. Ayant entendu sa confession et se rendant compte que sa situation est sans remède, le père Aubry ne peut que consoler la jeune femme: «vous perdez peu de chose, en perdant ce monde!» (p. 143) Dans une scène hautement symbolique, Atala est enterrée et le père Aubry renvoie Chactas à sa mère. L'épilogue, à la troisième personne, raconte les suites de l'histoire et le malheureux destin de ses personnages.

Ainsi dans Atala, la triste fin de l'héroïne est imputable à la mère, qui expie son propre péché charnel sur la tête de sa fille. Mais «l'ignorante Atala» (p. 105) aurait dû savoir qu'un tel vœu pouvait être annulé. C'est au père Aubry d'expliquer l'étiologie du mal d'Atala :

\footnotetext{
Ma fille, répondit le bon religieux, en versant des larmes, et les essuyant avec ses doigts tremblants et mutilés; ma fille, tous vos malheurs viennent de votre ignorance; c'est votre éducation sauvage et le manque d'instruction nécessaire qui vous ont perdue [...]. (p. 143 ; c'est moi qui souligne)
}

La bonté du prêtre, sa compassion, ses mains mutilées font du père Aubry une figure christique. Atala, pour sa part, incarne non seulement l'ignorance, mais sa face positive, l'innocence. Nonobstant la tragédie du vœu, il y a dans Atala une idéalisation de la virginité. À cet égard, la description du cadavre d'Atala, souvent commentée, est capitale. Éclairés par une lune qui se lève « comme une blanche vestale » (p. 153), Chactas et le père Aubry veillent la vierge. Ses joues sont « d'une blancheur éclatante », ses mains «d'albâtre » tiennent un crucifix d'ébène. "Je n'ai rien vu de plus céleste », dit Chactas; "Quiconque eût ignoré que cette jeune fille avait joui de la lumière, aurait pu la prendre pour la statue de la Virginité endormie. » (p. 153) Quand, plus tard, Chactas jette sur le corps « la terre du sommeil », il observe que son sein reste visible, « [surmontant] quelque temps le sol noirci, comme un lis blanc s'élève au milieu d'une sombre argile » (p. 154). Surdétermination remarquable, destinée à évoquer la pu-

12. Chateaubriand, Atala. René. Les Aventures du dernier Abencérage, Paris, Flammarion, 1996, p. 136. Toute référence ultérieure au roman sera empruntée à cette édition. 
reté virginale de la jeune victime, les nombreuses allusions au blanc suggèrent également le marmoréen : cette « statue de la Virginité endormie » aux « mains d'albâtre » sort de l'argile, œuvre d'art sculptée par Chateaubriand non seulement pour illustrer la bonté d'une Providence «qui conduit tout $\gg^{13}$, mais peut-être aussi pour des raisons plus égoïstes ${ }^{14}$.

Jean-Marie Roulin constate que les personnages féminins de Chateaubriand sont souvent «dot[és] d'attributs mariaux ${ }^{15} »$. Sans reprendre son travail d'analyse sur les sources de ce phénomène, bornons-nous à signaler que le culte de la virginité s'accompagne dans Atala d'un nombre extraordinaire d'allusions à la maternité. Comme c'est le cas pour la virginité, la maternité et la mort vont de pair, et l'ombre de la mère d'Atala plane sur ce récit, versant négatif de son alliée, la " Reine des Anges ».

Du blanc d'Atala au noir d'Ourika, la distance est vite parcourue. Au souci de pureté morale succède un souci de pureté raciale. Ourika, comme Atala, est hanté par le spectre de la virginité. Dans les deux cas, le personnage éponyme est destiné à mourir vierge en raison d'une opération de sauvetage qui se solde par un échec. Mais alors que la virginité semble idéalisée dans Atala, elle devient, dans Ourika, l'emblème de la solitude de l'héroïne qui aurait préféré mourir « sur le sein de sa mère », pour être ensuite enterrée dans sa terre maternelle, où « un peu de sable d'Afrique eût recouvert son corps » (p. 86). Triste écho du passage de l'enterrement dans Atala: la description du regret exprimé par Ourika oppose le sable à l'argile, le sein maternel au sein érotisé, la mort d'une enfant à celle d'une jeune femme. Mais le destin a prolongé la vie d'Ourika. À la femme transformée en statue dans Atala correspond une statue devenue femme dans Ourika: comme un objet d'art, sauvé de la destruction, rapporté du Sénégal et offert à une aristocrate dans un geste assimilé à une double animation («c'était me donner deux fois la vie » [p. 67]), Ourika sera comparée à la statue animée de Pygmalion: " et la fable ne nous dit pas si Galatée trouva le bonheur après avoir reçu la vie. » (ibid.) Toutefois, contrairement à Atala, Ourika doit son malheur non à l'ignorance mais à l'éducation.

Il n'y a, dans Ourika, aucune occurrence du mot « vierge » ou de ses dérivés, aucune allusion à la mère du Christ. Pourtant c'est bien à la virginité que l'exilée Ourika est condamnée par la couleur de sa peau, et le fait qu'elle termine sa vie dans un couvent qui appartient aux Ursulines est suggestif. La légende de sainte Ursule, selon laquelle la sainte aurait été livrée au martyre par les Huns en 383 avec 11000 vierges, était bien

13. Voir S. E. Mishriky, « Le thème de la providence dans Atala », Romance Notes, $\mathrm{n}^{\circ} 14$, 1972, p. 66-70.

14. Voir l'obséquieuse Épître dédicatoire au premier consul Bonaparte publiée en tête de l'édition du Génie du Christianisme, 1803, et cité dans C. Smethurst, Chateaubriand: "Atala » and "René », Londres, Grant \& Cutler, 1995, p. 11.

15. J.-M. Roulin, Chateaubriand, l'exil et la gloire, Paris, Champion, 1994, p. 76. 
connue au XIX ${ }^{\mathrm{e}}$ siècle. Comment ne pas voir de la part de Duras une intention malicieuse dans le choix de placer son héroïne dans un couvent dirigé par les Ursulines, dont la spécialité était l'éducation des jeunes filles? Le poids d'une vie chaste est également évoqué dans le prologue de l'histoire d'Ourika, car le médecin voit dans la virginité de la femme la source de son mal:

Je m'approchai timidement, car mon cœur s'était serré en voyant ces tombes, et je me figurais que j'allais contempler une nouvelle victime des cloîtres; [...] mon intérêt s'exaltait pour celle que j'allais visiter, en proportion du genre de malheur que je lui supposais. (p. 64; c'est moi qui souligne)

Le lecteur averti n'est guère dupe des euphémismes du médecin: la religieuse est celle qui n'a pas accompli sa «mission féminine » : la procréation. La marquise, elle non plus, ne conçoit pas le destin d'Ourika en dehors des normes du mariage et de la famille: "Que deviendra-t-elle? [...] à qui la marierez-vous? » (p. 71) Plus tard, lorsqu'elle rend visite à une Ourika languissante, elle présume que sa mélancolie a sa source dans la douleur d'un amour frustré :

Oui, Ourika, tous vos regrets, toutes vos douleurs ne viennent que d'une passion malheureuse, d'une passion insensée; et si vous n'étiez pas folle d'amour pour Charles, vous prendriez fort bien votre parti d'être négresse. (p. 93 ; c'est moi qui souligne)

À la passion illicite pour un « frère » déjà marié s'ajoute la folie hystérique qui est, au XIX ${ }^{\mathrm{e}}$ siècle, l'attribut de la femme. Prononçant ce diagnostic avec sa sécheresse coutumière, la marquise s'en va, déçue par le silence d'Ourika. Par sa structure, le discours de la marquise fait écho à celui du père Aubry dans Atala. Par son message et son ton, il s'y oppose, la sévérité de la marquise étant le contrepoint de la compassion touchante du prêtre. Le père Aubry cherche à innocenter Atala et à la consoler; la marquise culpabilise Ourika. Certes, la dernière phrase du roman semble indiquer de la part d'Ourika un amour coupable ${ }^{16}$. Pourtant, cet amour est loin d'être la source de toutes les douleurs et de tous les regrets d'Ourika. Mettant sa mélancolie entièrement sur le compte de la « passion insensée » qu'elle discerne chez Ourika, la marquise reflète-t-elle le racisme de la société européenne à l'égard de la lascivité des « sauvages », comme le voudrait Nathaniel Wing (op. cit., p. 90) ? La thèse est d'autant plus séduisante que le prêtre à qui Ourika se confesse par la suite la rassure sur la passion dont elle est accusée: « Votre cœur est pur, me dit-il. » (p. 94)

Si l'érotique n'entre pas dans les sentiments qu'Ourika a pour Charles, quelle est la source de sa mélancolie? Ceci précisément, qu'elle se sent de

16. « Laissez-moi aller, Charles, dans le seul lieu où il me soit permis de penser sans cesse à vous. » (p. 96) 
trop; elle n'a pas de raison d'être. Question de dignité humaine, elle cherche à se dévouer à autrui. C'est pour cela que les premières paroles de la marquise l'ont blessée si profondément: « j'avais besoin de ce que j'aimais, je ne songeais pas que ce que j'aimais n'avait pas besoin de moi. » (p. 73) Sa seule consolation réside dans ses rapports avec $\mathrm{M}^{\text {me }}$ de $\mathrm{B}$. et Charles: « [Je] ne pouvais en effet me rattacher à la vie, que par l'idée d'être nécessaire ou du moins utile à ma bienfaitrice. » (p. 74) La Terreur lui fournit la possibilité de soigner $\mathrm{M}^{\mathrm{me}}$ de $\mathrm{B}$. pendant un certain temps, mais, une fois la Révolution terminée, elle recommence à souffrir. Quand elle se voit remplacée dans la vie de Charles par Anaïs, son désespoir s'accroît. Le jour du mariage, elle a de nouveau « cet affreux sentiment de l'inutilité de l'existence » (p. 88). Son besoin de servir les autres se traduit par le désir d'être mère, même au prix de sa liberté:

\footnotetext{
Qu'avais-je fait à ceux qui crurent me sauver en m'amenant sur cette terre d'exil? Pourquoi ne me laissait-on pas suivre mon sort? Eh bien! je serais la négresse esclave de quelque riche colon [...] mais j'aurais $[\ldots]$ un compagnon de ma vie, et des enfants de ma couleur, qui m'appelleraient leur mère! (p. 90)
}

Mentalité d'esclave qui ne vit que pour servir les autres? Mentalité de femme pour qui le don de soi fait partie de sa psyché? C'est peut-être ce qu'on est censé croire. Toujours est-il que dans ce texte, la maternité est associée, non à la mort comme dans Atala, mais à la vie. Dans Atala, les liens familiaux sont une source de malheur; dans Ourika, ils sont perçus comme le seul bien. Ourika finira par trouver dans le couvent « une famille de choix » qui lui permettra d'être « [la] mère de tous les orphelins, la fille de tous les pauvres vieillards, la sœur de tous les malheureux » (p. 95).

Ainsi, beaucoup de symétries (positives et négatives) rapprochent Ourika d'Atala, suggérant la possibilité que Duras ait cherché dans son ouvrage à mettre en question les idées reçues qui sous-tendent celui de Chateaubriand. Cette intention ressort encore plus clairement quand on considère le rôle dévolu aux hommes. Chez Chateaubriand, les personnages masculins, instruments d'une Providence bienfaisante, sont héroïques. Il y a le «magnanime Simaghan » (p. 120) qui recueille une femme déshonorée (la mère d'Atala) et lui pardonne sa faute; le « généreux protecteur » Lopez (p. 96) qui sauve le jeune Chactas de l'esclavage; le « divin vieillard » (p. 149), Aubry, qui porte secours aux deux fuyards au milieu d'un orage pour les amener à sa mission et qui console par la suite une Atala mourante. Même le vieux narrateur Chactas en vient à personnifier une sagesse issue de la souffrance. Or, à tous ces mâles héroïques correspondent, dans le roman de Duras, des hommes qui, malgré leurs bonnes intentions, sont caractérisés par une impuissance fondamentale et surtout par un défaut de raison, censément le propre du mâle. Ainsi l'idéal chevaleresque, affirmé dans Atala par des personnages masculins qui incarnent la sagesse et l'altruisme, est profondément subverti dans Ourika. 
Considérons M. le chevalier de B., gouverneur du Sénégal et à ce titre représentant de l'autorité politique. De tous les personnages masculins, c'est le moins développé mais le plus important, car c'est le catalyseur de toute l'action du roman. Prenant en pitié une petite fille qu'on emporte à bord d'un négrier, il l'achète et la ramène en France pour la donner en cadeau à sa tante, « la personne la plus aimable de son temps » (p. 67). La " libération » n'en est pas une, car une forme d'esclavage peut en cacher une autre. Les actions attribuées au gouverneur (acheter, rapporter, donner) font d'Ourika un objet d'échange et ce statut n'évoluera pas. L'on ne peut pas pour autant douter de la compassion du gouverneur ni de la pureté de ses intentions. Tout au plus peut-on l'accuser d'imprévoyance. Et pourtant l'impasse de la vie d'Ourika découle de son geste. L'idéal du chevalier - sauver la demoiselle en détresse - subit chez $\mathrm{M}^{\mathrm{me}}$ de Duras une démystification, car le réel intervient. Malgré les généreux sentiments qui ont dicté son geste, l'incapacité du gouverneur à prévoir les éventuels résultats de son acte «philanthropique » est une erreur de jugement. Duras attaque ici « le cancer [des] bonnes intentions » (édition de Little, p. 56).

Suivant la chronologie du récit enchâssé, le deuxième personnage masculin à devenir la cible de Duras est Charles. À la différence du chevalier de B., ce personnage est longuement développé, et nous le suivons depuis son enfance jusqu'à sa maturité. Élevé par $\mathrm{M}^{\mathrm{me}}$ de B., Charles est, comme Ourika, orphelin de mère, mais contrairement à elle, il bénéficie de tous les avantages que lui confèrent sa masculinité, sa race et sa classe sociale. Ainsi, alors que la petite Ourika reste chez $\mathrm{M}^{\text {me }}$ de $\mathrm{B}$. à apprendre la peinture, le chant et les langues étrangères, Charles quitte la maison à l'âge de sept ans pour aller au collège; de là, il part en voyage pendant deux ans pour visiter l'Angleterre, l'Allemagne et l'Italie. De retour en février 1793, Charles s'occupe de $\mathrm{M}^{\mathrm{me}}$ de $\mathrm{B}$. jusqu'à la fin de la Terreur. C'est à ce moment-là qu'on lui propose un mariage avec une orpheline riche. À seize ans, «belle comme le jour » (p. 84), Anaïs de Thémines ne tarde pas à séduire Charles qui, pour sa part, se voit comme « le protecteur de cet ange ». Leur mariage est fertile: la naissance d'un fils (évidemment) comble leur bonheur et provoque, chez Ourika, une prise de conscience amère de sa propre solitude.

Mais c'est surtout dans ses rapports avec Ourika que le caractère de Charles se révèle. Détestant l'affectation, croyant à la justice, ému par la perspective de sauver une demoiselle en détresse (surtout quand elle est belle et riche), ne respectant que les douleurs « raisonnables », Charles semble à première vue incarner l'idéal aristocratique de la masculinité. Il n'y a que l'expérience militaire qui lui manque, mais sa jeunesse l'en excu$\mathrm{se}^{17}$. Pourtant, au retour de ses voyages d'apprentissage, celui qu'Ourika avait considéré comme son "protecteur, [son] conseil, et [son] soutien » 
(p. 68-69) ne la protège plus, ne lui donne plus de conseils, ne la soutient plus. Au contraire, il est incapable de reconnaître la souffrance d'Ourika, tant il est préoccupé de son propre bonheur. Dans toute sa conduite se manifestent ses préjugés contre sa « sœur » qui n'existe pas comme être humain à part entière, n'étant plus qu'une oreille passive et complaisante qu'il peut remplir de ses paroles. Alors qu'elle est caractérisée par un altruisme foncier, il est égoïste à l'extrême; alors qu'elle supporte en silence son chagrin, il lui parle de tout; alors qu'elle se mésestime, il respire la suffisance, convaincu qu'il peut être pour Anaïs « le père, la mère qu'elle a perdus [et aussi] son mari, son amant » (p. 86).

D'ailleurs, Ourika n'ignore pas les faiblesses de Charles : elle lui reconnaît le « défaut de voir [de l'affectation] où il n'y en avait pas » (p. 81); elle critique « [la] tournure un peu sévère de ses idées » (p. 82) et met en question ses théories sur la douleur (« qui peut dire ce que c'est que la raison? »[p. 82]). Manque de perspicacité, égoïsme, jugement douteux: Charles n'incarne en rien les qualités propres au galant homme tel que les stéréotypes de l'époque le définissaient. Pourtant pour Ourika cette amitié déséquilibrée, si peu satisfaisante qu'elle soit, reste un bien, car elle lui prouve qu'on a besoin de sa présence. C'est seulement quand elle se voit remplacée par Anaïs qu'elle sombre dans une profonde mélancolie.

Comme dans Atala, où le bon père Aubry doit essayer de réparer le mal fait par son confrère, « l'imprudent missionnaire » (p. 143) qui dirigeait la mère d'Atala, les représentants de la religion dans Ourika forment un groupe hétérogène, à cette différence près que dans Atala c'est « le prêtre divin » (p. 131) qui domine, alors que dans Ourika les prêtres ne sont qu'humains. Le «prêtre respectable» (p. 74) qui prépare Ourika pour sa première communion ne lui explique pas bien le concept de piété; le « saint vieillard $»($ p. 74) qui est son confesseur ne soupçonne pas sa souffrance; le vieil abbé qui fait partie de la société de $\mathrm{M}^{\mathrm{me}}$ de $\mathrm{B}$. pendant la Terreur raisonne mal, se faisant «un enchaînement si complet d'idées fausses [qu'il soutient] avec tant de bonne foi » qu'il devient pour $\mathrm{M}^{\text {me }}$ de B. « une source inépuisable d'amusement » (p. 81). Il n'y a que le prêtre appelé à administrer à Ourika le viatique qui joue un rôle bénéfique, car il la déclare innocente d'un amour coupable pour son « frère » et il l'amène à Dieu, ses « paroles simples » (p. 94) lui apportant une paix longtemps recherchée.

Reste le médecin. Si, en choisissant comme premier narrateur un homme qui par devoir professionnel est objectif et fiable, Duras ne fait que suivre une convention bien établie, la façon dont elle le caractérise fait douter de sa neutralité. Sa jeunesse et son inexpérience sont évoquées par l'incipit, car il est « arrivé depuis peu de mois de Montpellier », ville connue pour sa faculté de médecine, la plus ancienne de la France. Étant donné une allusion antérieure à un certain docteur Barthez qui est venu soigner Ourika («[il] m'examina avec soin, me tâta le pouls et dit brusquement que je n'avais rien » [p. 73]), cette évocation de Montpellier est 
importante. Paul-Joseph Barthez (1734-1806), qui enseignait à la faculté de médecine de l'université de Montpellier, était célèbre pour sa théorie du principe vital. Selon ce principe, qui s'opposait aux théories mécanistes et matérialistes, le corps se composait non seulement de matière, mais aussi d'un principe vital qui animait la matière inerte du corps. Ce n'était pas à l'âme, concept théologique, que Barthez pensait en élaborant sa théorie, et pourtant la curieuse remarque faite par le médecin anonyme du cadre narratif, appelé à « sauver» Ourika (« l'âme vivait encore, mais le corps était détruit» [p. 64]), semble faire allusion au « vitalisme » de l'école de Montpellier. Son adhésion à une théorie approuvée par l'Église n'implique pas pour autant une approbation de ses pratiques, et le discours anticlérical de l'époque révolutionnaire trouve un écho dans son horreur de la vie cloîtrée. Devant une religieuse rongée par la mélancolie, il va droit au diagnostic: c'est le célibat qui la tue ${ }^{18}$. Il manifeste également un préjugé typique de son époque à l'égard des Noirs: sa surprise en voyant une Négresse s'augmente quand il l'entend parler, car il ne s'attend pas à « la politesse de son accueil et le choix des expressions dont elle se servait» (p. 64). L'étonnement du médecin le trahit: malgré certaines idées révolutionnaires, il est très "Ancien Régime » dans son incapacité à reconnaître une noblesse autre que celle conférée par la naissance. Mais c'est avant tout par l'inefficacité de son traitement que le médecin forme le dernier maillon dans la chaîne des mâles impuissants mis en scène par Duras. Sur son traitement proprement médical, le texte reste vague. Nous savons seulement qu'il " parut produire quelque effet » (p. 65) quand Ourika se décida enfin à céder à ses prières en lui racontant son histoire pour qu'il puisse " guérir [le passé] ». Une lecture anachronique de ce roman évoquerait la cure par la parole de Freud; mais à la fin du XVIII ${ }^{\mathrm{e}}$ siècle, cette méthode n'avait pas encore été élaborée et rien ne nous permet d'accorder du crédit au remède proposé par le médecin, d'autant plus qu'il s'oppose à celui, plus réussi, de $\mathrm{M}^{\text {me }}$ de B. qui, craignant, selon Ourika, «d'exalter [s]a peine en [lui] permettant de la confier » (p. 74), préfère distraire la malade en l'occupant de ses peines à elle.

Vue sous cet angle, la fameuse «raison » du médecin se révèle défectueuse, sa méthode étant d'ailleurs incompatible avec les premiers conseils qu'il donne à Ourika « de calmer son imagination, de se distraire, d'éloigner des sentiments pénibles » (p. 64). En la poussant à raconter son histoire, tout en admettant d'emblée qu'il la trouvera "déraisonnable», il joue un rôle parallèle à celui de la marquise qui demande à Ourika de lui ouvrir son cœur (" personne ne prend à vous plus d'intérêt que moi » [p. 92]). Dans les deux cas, on soupçonne un certain voyeurisme. Il va sans dire que ce voyeurisme est bien motivé sur le plan textuel: sans la narration de l'héroïne, il n'y aurait pas de nouvelle. Et pourtant, Duras

18. Voir S. Desan, The Family on Trial in Revolutionary France, Berkeley, University of California Press, 2004, p. 32, 112 et 353. 
aurait pu faire d'Ourika l'auteure d'un journal intime. Si elle ne l'a pas fait, c'est, d'une part parce qu'elle voulait préserver l'oralité qui a été une des caractéristiques marquantes du récit de Chateaubriand, et d'autre part parce que le personnage du médecin était essentiel à la transmission de son message sur la vanité des hommes. Ourika elle-même est sceptique quant au remède proposé. À l'observation du médecin («ce passé, je ne puis le guérir sans le connaître » [p. 64]), elle réplique: «Hélas ! ce sont des folies!» (p. 64) Parle-t-elle des événements de sa vie ou des idées du médecin? L'ambiguïté est voulue.

Dans Ourika, comme dans Atala, la femme - voilée quand on la voit pour la première fois - est l'objet du regard masculin. Mais alors que dans Atala, la femme est érotisée par ce regard et qu'elle reste séduisante, vivante jusque dans la mort, dans Ourika, le regard, clinique, la pathologise, la voue à la mort alors qu'elle est toujours en vie. « La mort avait marqué sa victime » (p. 65), constate le médecin d'Ourika, en la rencontrant pour la première fois. Façon d'excuser à l'avance l'échec de son traitement. Peut-on guérir le passé ? Pas plus que les révolutionnaires, en tranchant des têtes, ne pouvaient redresser les torts de l'Ancien Régime.

Si les hommes ne sont pas du côté de la raison dans Ourika, qui donc incarne cette faculté de penser? En premier lieu, $\mathrm{M}^{\mathrm{me}}$ de B. : « L'idée la plus ordinaire devenait féconde si elle passait par la bouche de $\mathrm{M}^{\mathrm{me}}$ de $\mathrm{B}$.; son esprit et sa raison savaient la revêtir de mille nouvelles couleurs. » (p. 80) $\mathrm{Si}$ antipathique qu'elle soit, la marquise, «personne d'une raison froide, d'un esprit tranchant » (p. 70), incarne la dureté de la logique. Ourika ellemême, dont l'intelligence ne laisse pas de doute, analyse sa situation avec une lucidité navrante.

Dans une étude perspicace sur le roman romantique, The Male Malady: Fictions of Impotence in the French Romantic Novel, Margaret Waller fait remarquer que les auteurs masculins, en créant des héros féminisés (tels René et Adolphe) fournissent une nouvelle version de la masculinité qui modifie d'une manière importante l'image traditionnelle de l'homme viril mise en place dès l'époque des guerres napoléoniennes. Selon l'hypothèse de Waller, cependant, la prétendue impuissance de ces héros frustrés, mélancoliques, paralysés par l'inaction et vivant en marge de la société, est une ruse: le héros exploite ses «faiblesses» pour maintenir son pouvoir sur la femme. Considéré dans cette perspective, le «mal du siècle » est une maladie spécifiquement masculine, destinée à garantir la survie d'une société patriarcale où la femme reste soumise à l'homme.

L'hégémonie masculine est intacte dans Ourika. Claire de Duras ne « féminise » pas ses personnages masculins et leur laisse le pouvoir (politique, social, religieux, médical) dont ils bénéficiaient dans la société de son temps. Ce qu'elle leur enlève, par contre, c'est l'intelligence qui pourrait légitimer ce pouvoir et qui, selon les stéréotypes de l'époque, était l'apanage de l'homme. Cette intelligence, elle la transfert aux femmes. 
Les hommes, en revanche, pleins de bonne volonté, mus par un idéal chevaleresque suranné, sont caractérisés par une insuffisance intellectuelle qui se manifeste de diverses façons: imprévoyance, aveuglement, inconséquence, incompétence. De par ces défauts qui les opposent aux sages mis en scène dans Atala, ils sont non seulement incapables de jouer le rôle qui devrait être le leur - celui de sauver la demoiselle en détresse -, mais ils deviennent les instruments mêmes de sa souffrance. Duras met au jour le caractère fallacieux des stéréotypes masculins de son époque. Qu'il soit représenté comme bienfaiteur, confident, diagnostiqueur, guérisseur ou guide spirituel, l'homme n'est qu'un cabotin, incapable de bien jouer son rôle. Par sa destruction du mythe culturel d'une masculinité héroïque, Ourika est un roman profondément contestataire ${ }^{19}$.

Mary Donaldson-Evans Université du Delaware, États-Unis

19. Je tiens à remercier Lance Donaldson-Evans et Barbara T. Cooper pour leurs conseils. 